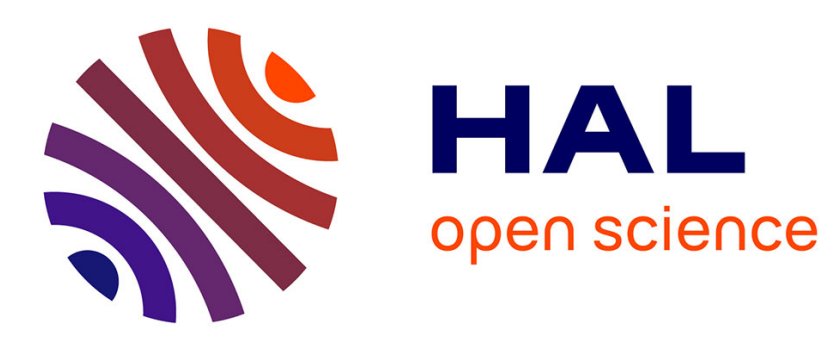

\title{
Dynamic-weighted ensemble for fatigue crack degradation state prediction
}

Hoang-Phuong Nguyen, Jie Liu, Enrico Zio

\section{To cite this version:}

Hoang-Phuong Nguyen, Jie Liu, Enrico Zio. Dynamic-weighted ensemble for fatigue crack degradation state prediction. Engineering Fracture Mechanics, 2018, 194, pp.212-223. 10.1016/j.engfracmech.2018.03.013 . hal-01988976

\section{HAL Id: hal-01988976 https://hal.science/hal-01988976}

Submitted on 22 Jan 2019

HAL is a multi-disciplinary open access archive for the deposit and dissemination of scientific research documents, whether they are published or not. The documents may come from teaching and research institutions in France or abroad, or from public or private research centers.
L'archive ouverte pluridisciplinaire HAL, est destinée au dépôt et à la diffusion de documents scientifiques de niveau recherche, publiés ou non, émanant des établissements d'enseignement et de recherche français ou étrangers, des laboratoires publics ou privés. 


\title{
Dynamic-weighted ensemble for fatigue crack degradation state prediction
}

\author{
Hoang-Phuong Nguyen ${ }^{\mathrm{a}}$, Jie Liu ${ }^{\mathrm{b}, \mathrm{c}}$, Enrico Zio ${ }^{\mathrm{a}, \mathrm{c}, \mathrm{d} \text {,* }}$ \\ ${ }^{\text {a } C h a i r ~ o n ~ S y s t e m ~ S c i e n c e ~ a n d ~ t h e ~ E n e r g e t i c ~ C h a l l e n g e, ~ E D F ~ F o u n d a t i o n, ~ C e n t r a l e S u p e ́ l e c, ~ U n i v e r s i t e ́ ~ P a r i s-S a c l a y, ~} 9$ rue Joliot-Curie, 91192 Gif-sur- \\ Yvette, France \\ ${ }^{\mathrm{b}}$ School of Reliability and Systems Engineering, Beihang University, 37 Xueyuan Road, Haidian, Beijing, China \\ ' Sino-French Risk Science and Engineering (RISE) Laboratory, Beihang University, 37 Xueyuan Road, Haidian, Beijing, China \\ d Dipartimento di Energia, Politecnico di Milano, Via La Masa 34, 20156 Milano, Italy
}

\section{A R T I C L E I N F O}

\section{Keywords:}

Prognostics and Health Management (PHM)

Recursive Bayesian

Dynamic ensemble

Multiple stochastic degradation

Fatigue crack growth

\begin{abstract}
A B S T R A C T
This paper proposes a prognostic framework for online prediction of fatigue crack growth in industrial equipment. The key contribution is the combination of a recursive Bayesian technique and a dynamic-weighted ensemble methodology to integrate multiple stochastic degradation models. To show the application of the proposed framework, a case study is considered, concerning fatigue crack growth under time-varying operation conditions. The results indicate that the proposed prognostic framework performs well in comparison to single crack growth models in terms of prediction accuracy under evolving operating conditions.
\end{abstract}

\section{Introduction}

Cracks are among the most common degradations in equipment of several major industries, including manufacturing [1,2], construction [3,4], aerospace [5-7], automotive [8,9], energy [10,11], etc. A study conducted by the American Society of Civil Engineers (ASCE) [4] has revealed that more than $80 \%$ of the collapses of American bridges in steel were caused by fatigue and fracture in structural elements. In [5], it has been shown that in aerospace industry, cracks develop in most critical components of rotorcrafts, such as the main rotor blade, the major cabin frame cap splice, and the tail boom. These unexpected degradations increase the operation risk and can cause severe economic losses in case of breakdowns [12-15]. Thus, for the past several decades, the development of reliable prognostic systems to accurately analyze and estimate the crack propagation in an equipment has attracted the attention of industrial practitioners and researchers.

Some prognostic models have been developed using historical degradation data from a population of similar equipment, whereas the real-time condition monitoring data of the specific equipment were not considered [16-19]. These historical information, however, may not be always available in practical industrial systems, especially for newly produced equipment or expensive components where the data acquisition costs too much [20]. More importantly, different practical operational conditions, such as load, temperature, and speed, could significantly impact on the rate of the degradation processes, which makes each specific system present a particular degradation trajectory [21]. Therefore, it is important to include the condition monitoring data of the targeted equipment. To address this issue, Cadini et al. [14] introduced a failure prognostic method for fatigue crack growth prediction using a stochastic crack growth model and a Bayesian technique to dynamically update the degradation state from a sequence of monitored measurements. In this sense, recursive Bayesian algorithms are potentially suitable for model-based prognostic frameworks. Indeed,

\footnotetext{
* Corresponding author at: Chair on System Science and the Energetic Challenge, EDF Foundation, CentraleSupélec, Université Paris-Saclay, France E-mail addresses: hoang-phuong.nguyen@centralesupelec.fr (H.-P. Nguyen), liujie805@buaa.edu.cn (J. Liu), enrico.zio@centralesupelec.fr (E. Zio).
} 


\begin{tabular}{|c|c|c|c|}
\hline \multirow{2}{*}{\multicolumn{2}{|c|}{ Nomenclature }} & $\mathrm{t}$ & time (cycle) \\
\hline & & $\mathrm{T}$ & prediction time (cycle) \\
\hline \multicolumn{2}{|c|}{ Abbreviations } & $\mathrm{w}$ & $\begin{array}{l}\text { weight of individual degradation model in the } \\
\text { ensemble }\end{array}$ \\
\hline ASCE & American Society of Civil Engineers & $\mathrm{x}$ & degradation state $(\mathrm{mm})$ \\
\hline FDI & Fault Detection and Isolation & $\widehat{\mathbf{x}}$ & estimated degradation state of individual de- \\
\hline IMMPF & Interacting Multiple Model Particle Filter & & gradation model $(\mathrm{mm})$ \\
\hline MSE & Mean Square Error & $\widetilde{\mathbf{x}}$ & estimated degradation state of the ensemble (mm) \\
\hline MLE & Maximum Likelihood Estimation & $\mathrm{y}$ & augmented degradation state \\
\hline PDF & Probability Density Function & $\mathrm{z}$ & measurement (mm) \\
\hline PHM & Prognostics and Health Management & & \\
\hline SIF & Stress Intensity Factor & \multicolumn{2}{|c|}{ Greek symbols } \\
\hline \multicolumn{2}{|c|}{ Latin symbols } & $\delta$ & $\begin{array}{l}\text { time horizon for error coefficient estimation (cy- } \\
\text { cles) }\end{array}$ \\
\hline $\mathrm{C}$ & material constant & $\Delta K$ & stress intensity factor $(\mathrm{MPa} \sqrt{\mathrm{m}})$ \\
\hline f & state transition function & $\Delta \sigma$ & cyclic stress amplitude (MPa) \\
\hline $\mathrm{F}$ & augmented state transition function & $\Delta t$ & time interval (cycle) \\
\hline$g$ & measurement function & $\theta$ & degradation model parameter \\
\hline G & augmented measurement function & $\sigma_{\omega}^{2}$ & state noise variance \\
\hline$h(x)$ & geometric factor & $\sigma_{v}^{2}$ & measurement noise variance \\
\hline $\mathrm{m}$ & material constant & $\tau$ & prediction interval (cycle) \\
\hline $\mathrm{N}$ & number of fatigue load cycles (cycle) & $v$ & measurement noise \\
\hline $\mathrm{N}_{\mathrm{M}}$ & number of degradation models & $\varphi$ & estimation error coefficient of individual de- \\
\hline $\mathrm{p}$ & constant of polynomial crack growth model & & gradation model \\
\hline $\mathrm{R}$ & stress ratio of the crack growth process & $\omega$ & state noise \\
\hline
\end{tabular}

the prior distribution of the degradation states can be combined with the likelihood of the monitored measurements for updating the posterior distribution of the states adaptively when new measurements are available. In [22], Boris et al. presented a prognostic method based on a Bayesian technique to dynamically update the stress intensive range of the physical degradation model at each load cycle until failure, using the condition monitoring measurements. In another study, a comprehensive architecture for both fault detection and isolation (FDI), and failure prognosis for a UH-60 planetary carrier plate was carried out by exploiting a non-linear degradation model and a Bayesian variant, to effectively detect abnormal conditions and predict online the crack depth evolution of the equipment [23].

In practice, the performance of online prognostic models for fatigue crack growth heavily depends on the adopted physics-offailure model and it is very important to figure out an appropriate modelling framework for a specific degradation process under time-varying operation conditions. To address this issue, numerous fatigue crack growth models have been extensively studied [24-30]. In [31], a comparison of stochastic fatigue crack growth models including the Markov chain model, the Yang's power law model, and a polynomial model were carried out. The results showed that each degradation model has its own range of applicability, and only fits a certain particular degradation process. To the knowledge of the authors, there is no general consensus on a comprehensive prognostic model for fatigue crack growth under different degradation processes. Recently, in the applications of Lithiumion battery prognostics, hybrid and multi-degradation model ensembles have gained interest because of higher accuracy and better generalization capability than individual degradation models [32,33]. The basic idea behind these empirical frameworks is to find a set of diverse degradation models which cover different situations so that they complement each other. In [33], an interacting multiple model particle filter (IMMPF) was introduced to combine the estimations from three battery capacity degradation models. The study concluded that the interacting multiple model can achieve higher robustness in terms of smaller estimation errors and more stable performance than a single model.

In this paper, a prognostic framework for fatigue crack growth is proposed by integrating a recursive Bayesian technique and a dynamic ensemble. The degradation state of the component is estimated based on the condition monitoring data collected until the current load cycle, and short-term degradation state prediction is performed to anticipate and proactively prevent sudden breakdowns of the component in a near future. The key contribution of the work is the dynamic ensemble which combines different crack evolution models with dynamic weights. The dynamic weights are computed based on the historical estimation error for a predefined number of the latest load cycles. To the authors' knowledge, this ensemble framework has been here developed and applied for the first time for a prognostic problem of fatigue crack growth. To validate the performance of the proposed framework, a case study concerning fatigue crack growth with evolving operation conditions is carried out and the results are compared with those obtained by applying single degradation models.

The rest of this paper is organized as follows. Section 2 introduces the degradation models for fatigue crack growth and details the proposed prognostic framework. Section 3 describes the illustrative case study of fatigue crack growth with different load conditions. Finally, Section 4 concludes the study. 


\section{Proposed prognostic framework}

Fig. 1 illustrates an overall flow diagram of the proposed prognostic framework, which consists of the following two main stages: current degradation state estimation based on a recursive Bayesian technique and dynamic-weighted ensemble for prediction. More details about the proposed prognostic framework are described in the following sections.

\subsection{Recursive Bayesian technique for state estimation and parameter identification}

\subsubsection{State estimation with fixed parameters}

In this section, we first describe the technique for estimating the degradation state at time $t\left\{x_{t}, t \in \mathrm{N}\right\}$, given the available condition monitoring measurements $\left\{z_{t}, t \in \mathrm{N}\right\}$. We consider a state-space model describing the evolution of the degradation state $x_{t}$ and the measurement $z_{t}$ at the inspection time $t$, given by:

$$
\begin{aligned}
& x_{t}=f_{t}\left(x_{t-1}, \omega_{t-1}\right) \\
& z_{t}=g_{t}\left(x_{t}, v_{t}\right),
\end{aligned}
$$

where $f_{t}$ denotes the state transition function and $g_{t}$ is the measurement function; $\omega_{t-1}$ and $v_{t}$ are independent identically distributed (i.i.d.) state noise and measurement noise sequences, respectively. The current degradation state $x_{t}$ depends only on the previous state $x_{t-1}$, and not on the states at previous times. This represents a first-order Markov process with independent degradation increments. The Bayesian technique can be used for estimating the system state $x_{t}$ at time $t$ by constructing the posterior probability density function (pdf) $p\left(x_{t} \mid z_{1: t}\right)$ based on the state transition function and the monitored measurements. More specifically, the system state is recursively estimated by performing the following two steps, namely prediction and update.

The prediction step involves using both the previous state estimation $x_{t-1}$ and the state transition model in Eq. (1) to estimate the prior distribution of the current state $x_{t}$ via the Chapman-Kolmogorov equation, as follows:

$$
p\left(x_{t} \mid z_{1: t-1}\right)=\int p\left(x_{t} \mid x_{t-1}, z_{1: t-1}\right) p\left(x_{t-1} \mid z_{1: t-1}\right) d x_{t-1}=\int p\left(x_{t} \mid x_{t-1}\right) p\left(x_{t-1} \mid z_{1: t-1}\right) d x_{t-1},
$$

where $p\left(x_{t} \mid x_{t-1}\right)$ is the conditional probability distribution defined by the state model in Eq. (1). The initial distribution $p\left(x_{0} \mid z_{0}\right)=p\left(x_{0}\right)$ is assumed to be available and is known as the prior. In the update step, the new measurement $z_{t}$ is used for obtaining the posterior state pdf $p\left(x_{t} \mid z_{1: t}\right)$ by applying Bayes' theorem, as follows:

$$
p\left(x_{t} \mid z_{1: t}\right)=\frac{p\left(x_{t} \mid z_{1: t-1}\right) p\left(z_{t} \mid x_{t}\right)}{p\left(z_{t} \mid z_{1: t-1}\right)},
$$

where $p\left(z_{t} \mid x_{t}\right)$ is the likelihood function defined by the measurement model in Eq. (2) and $p\left(z_{t} \mid z_{1: t-1}\right)$ is a normalizing constant which

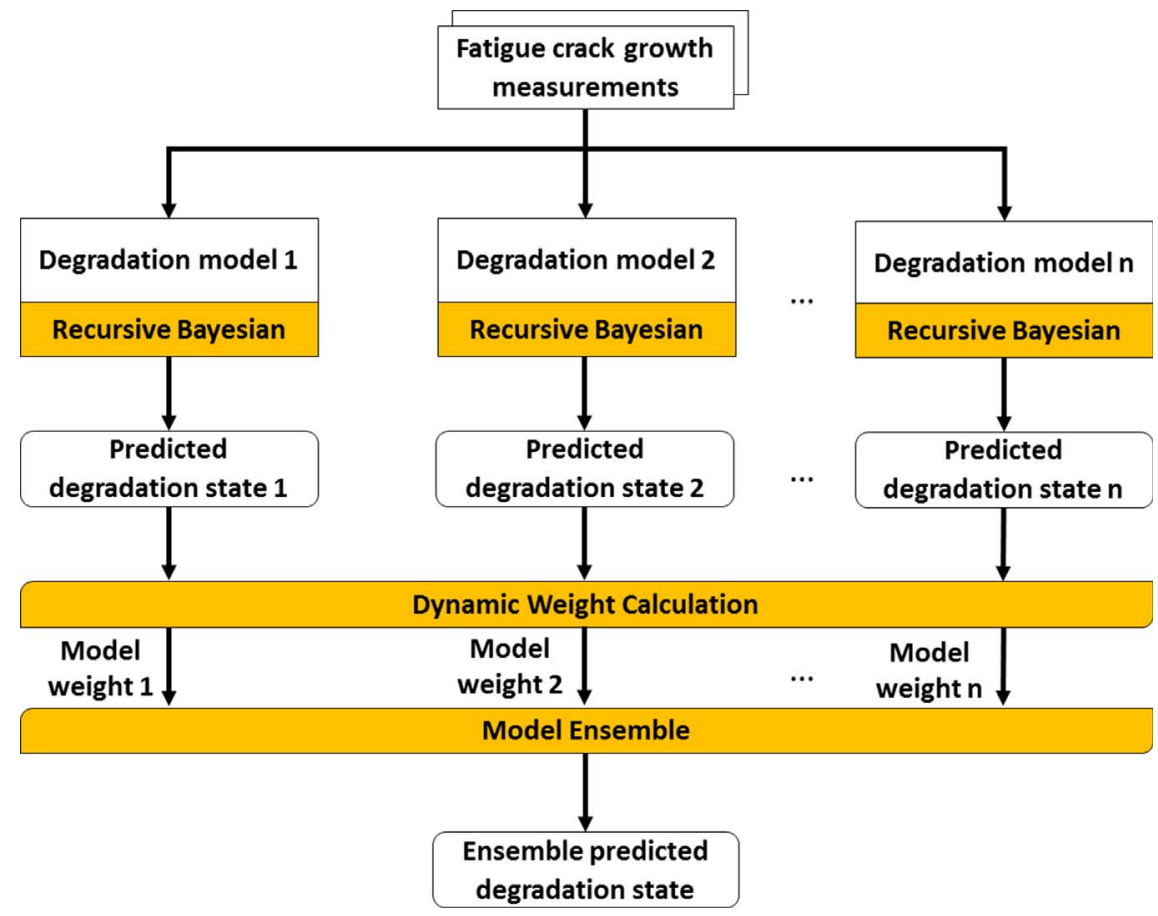

Fig. 1. Flow diagram of the proposed prognostic framework. 
is defined by:

$$
p\left(z_{t} \mid z_{1: t-1}\right)=\int p\left(x_{t} \mid z_{1: t-1}\right) p\left(z_{t} \mid x_{t}\right) d x_{t} .
$$

\subsubsection{Sequential parameter identification}

A main advantage of the proposed prognostic framework lies also in the capability of identifying time-varying parameters from the sequential data, as detailed in this section. Let us add the time-varying parameter $\theta_{t}$ to the state vector at the inspection time $t$, as follows:

$$
y_{t}=\left[\begin{array}{c}
x_{t} \\
\theta_{t}
\end{array}\right]
$$

For this new state vector, the state-space system model in Eqs. (1) and (2) can be reformulated as follows:

$$
\begin{aligned}
& y_{t}=F\left(y_{t-1}, \omega_{t-1}\right) \\
& z_{t}=G_{t}\left(y_{t}, v_{t}\right),
\end{aligned}
$$

where

$$
F(y, \omega)=\left[\begin{array}{c}
f(x, \omega) \\
\theta
\end{array}\right]
$$

and

$$
G_{t}(y, v)=g_{t}(x, v)
$$

Based on the modified model, the posterior joint distribution $p\left(y_{t} \mid z_{1: t}\right)=p\left(x_{t}, \theta_{t} \mid z_{1: t}\right)$ can be obtained by employing the recursive Bayesian algorithm discussed in the previous section. In this circumstance, the initial distribution is assumed independent of $\theta$, that is, $p\left(y_{0}\right)=p\left(x_{0}, \theta_{0}\right)=p\left(x_{0}\right)$. Finally, the marginal posterior distributions of the system state $x_{t}$ and the time-varying parameter $\theta_{t}$ can be obtained by:

$$
\begin{aligned}
& p\left(x_{t} \mid z_{1: t}\right)=\int p\left(y_{t} \mid z_{1: t}\right) d \theta_{t}=\int p\left(x_{t}, \theta_{t} \mid z_{1: t}\right) d \theta_{t} \\
& p\left(\theta_{t} \mid z_{1: t}\right)=\int p\left(y_{t} \mid z_{1: t}\right) d x_{t}=\int p\left(x_{t}, \theta_{t} \mid z_{1: t}\right) d x_{t}
\end{aligned}
$$

\subsubsection{Short-term state prediction}

We consider the degradation state estimation not only at the current load cycle $t$, i.e. state regression, but also the prediction at fixed prediction horizons $T=t+\tau$, i.e. short-term prediction. In the following case study, three scenarios are considered: (1) degradation state regression at the current time $T=t$; (2) short-term state prediction at time $T=t+100$; and (3) short-term state prediction at time $T=t+300$.

\subsection{Fatigue crack growth models}

For applying the Bayesian recursive technique for state estimation to the fatigue crack growth process, four stochastic degradation models of fatigue crack growth are considered, including Paris-Erdogan, polynomial, global function-based, and curve fitting technique-based models.

\subsubsection{Paris-Erdogan model}

One well known degradation model for fatigue crack growth is the Paris-Erdogan law [34], which represents the dependence between the crack growth rate $d x / d N$ and the Irwin's stress intensity factor $\Delta K$ [35] as follows:

$$
\frac{d x}{d N}=C(\Delta K)^{m}
$$

where $x$ is the crack length, $C$ and $m$ are material constants, and $N$ is the number of fatigue load cycles. In this study, the ParisErdogan model is employed for considering an infinite plate with a center crack subjected to a sinusoidally applied stress $\sigma$, where the geometric factor is equal to 1 and the stress intensity factor $\Delta K$ is defined as follows [35]:

$$
\Delta K=\Delta \sigma \sqrt{\pi x},
$$

where $\Delta \sigma$ is the cyclic stress amplitude.

To take into consideration the statistical variability of the crack growth rate in practice, Myötyri et al. [36] introduced a stochastic variant of the Paris-Erdogan model by using a process intrinsic stochasticity, given by:

$$
\frac{d x}{d N}=e^{\omega} C(\Delta K)^{m}
$$


where $\omega \sim N\left(0, \sigma_{\omega}^{2}\right)$ is an additional white Gaussian noise. For $\Delta t$ sufficiently small, the state-space model in Eq. (15) can be discretized as follows:

$$
x_{t}=x_{t-1}+e^{\omega} C(\Delta K)^{m} \Delta t,
$$

which represents a non-linear Markov process with independent evolution of the degradation state $x$.

\subsubsection{Polynomial model}

A mismatch of crack growth models based on power function with the median crack growth curve has been discovered [31,37]. To address this issue, a crack growth model based on polynomial function was proposed as follows [31]:

$$
\frac{d x}{d N}=e^{\omega}\left(p_{0}+p_{1} x+p_{2} x^{2}\right)
$$

where $p_{i}, i=0, \ldots, 2$ are polynomial constants. The stress intensity factor $\Delta K$ is not considered in this model [31]. In other studies, the polynomial model was shown to yield the best fit in the linear least square stage of the degradation process [32,33].

The Markov process representation for a polynomial function-based crack growth model can be defined as follows:

$$
x_{t}=x_{t-1}+e^{\omega}\left(p_{0}+p_{1} x+p_{2} x^{2}\right) \Delta t .
$$

\subsubsection{Global function}

Despite the fact that the Paris-Erdogan model and polynomial model show satisfactory performance in the description of the fatigue crack growth process, the effects of equipment geometry on the degradation process have not been taken into consideration [30]. To tackle this shortcoming, Hossien et al. [30] introduced a global function by further reformulating the model for stress intensity range in Eq. (14), using a geometric factor defined as follows:

$$
\Delta K=h(x) \Delta \sigma \sqrt{\pi x},
$$

where $h(x)$ is the geometric factor. In this work, a case study considering a center-cracked plate under uniform tension is considered for validating the global function-based crack growth model, in which the geometric factor $h(x)$ is defined by [38]:

$$
h(x)=1+0.128\left(\frac{x}{w}\right)-0.288\left(\frac{x}{w}\right)^{2}+1.523\left(\frac{x}{w}\right)^{3}
$$

where $w$ is the specimen width.

The global function-based crack growth model can be further discretized as follows:

$$
x_{t}=x_{t-1}+e^{\omega} C\left[1+0.128\left(\frac{x}{w}\right)-0.288\left(\frac{x}{w}\right)^{2}+1.523\left(\frac{x}{w}\right)^{3}\right]^{m}(\Delta \sigma \sqrt{\pi x})^{m} \Delta t .
$$

\subsubsection{Curve fitting function}

Still in [30], the authors presented another empirical crack growth model based on a curve fitting technique, given by:

$$
\frac{d x}{d N}=e^{\omega}\left(\frac{1}{C_{1} x^{m}+C_{2}}\right)
$$

where $C_{1}, C_{2}, m$ denote the model constants, whose values need to be estimated. According to [30], the stress intensity factor $\Delta K$ is not considered in the model.

The authors showed that the crack growth model based on the curve fitting function had capabilities of outperforming the conventional models, such as the power function and the polynomial function, in terms of higher prediction accuracy and lower computation cost. The discretized Markov process representation for a curve fitting function-based crack growth model can be defined as follows:

Table 1

Detailed settings of model characteristics of fatigue crack growth case studies.

\begin{tabular}{ll}
\hline State noise variance & $\sigma_{\omega}^{2}=0.49$ \\
Measurement noise variance & $\sigma_{v}^{2}=0.16$ \\
& $\cdot C=0.1$ \\
Paris-Erdogan model & $\cdot m=1.3$ \\
& $\cdot p_{0}=1.4 \times 10^{-3}, p_{1}=1.5 \times 10^{-3}, p_{2}=1 \times 10^{-5}$ \\
Polynomial model & $\cdot C=0.005$ \\
Global function-based model & $\cdot m=0.245$ \\
& $\cdot w=1 \mathrm{~mm}$ \\
Curve fitting function-based model & $\cdot C_{1}=250, C_{2}=0.3$ \\
& $\cdot m=-0.7$ \\
\hline
\end{tabular}




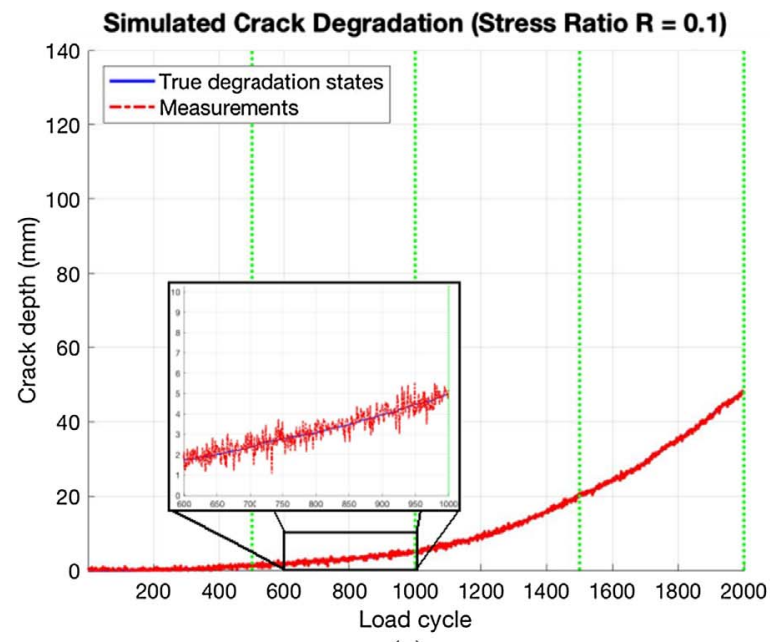

(a)

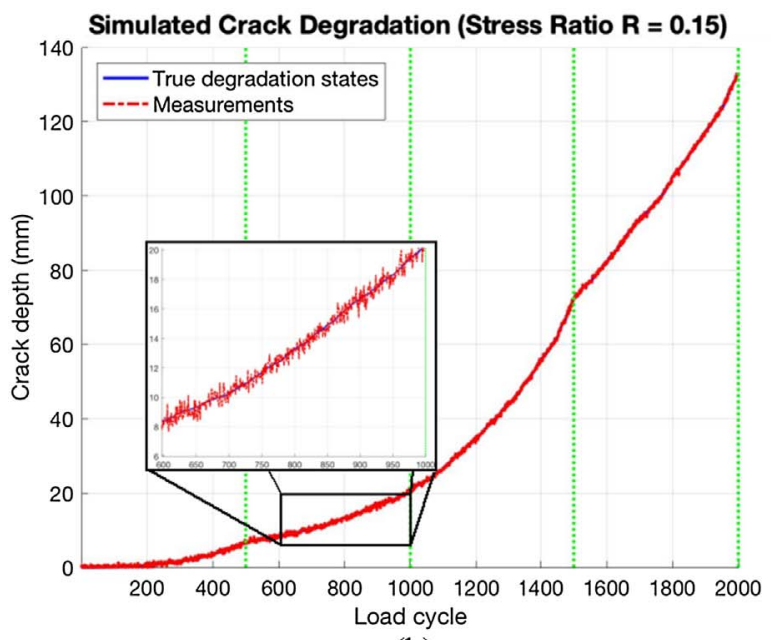

(b)

Fig. 2. Simulated crack depth evolution profiles with different load ratios (a) $R=0.1$ and (b) $R=0.15$.
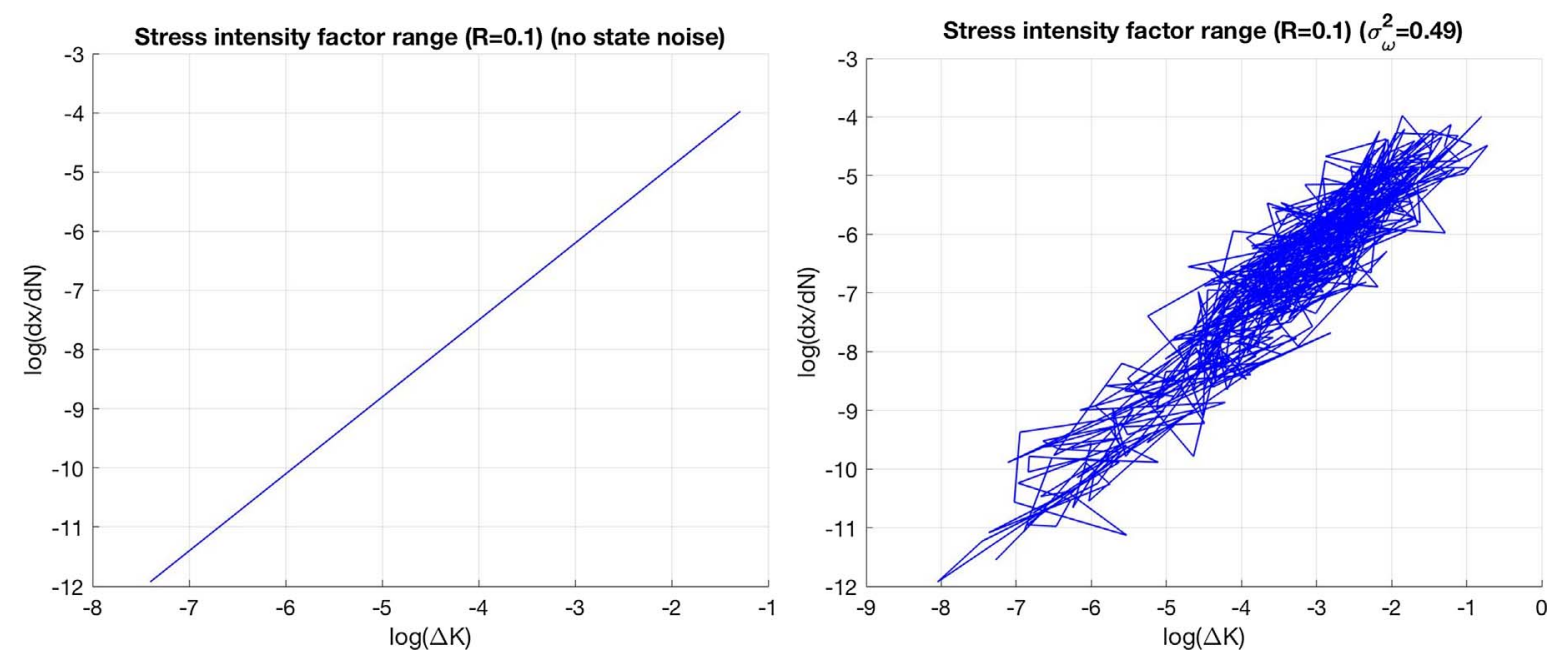

Fig. 3. Stress intensity factor range, $\Delta K \log$ scale, with $R=0.1$. Without state noise considered (left) and with state noise $\sigma_{\omega}^{2}=0.49$ (right).
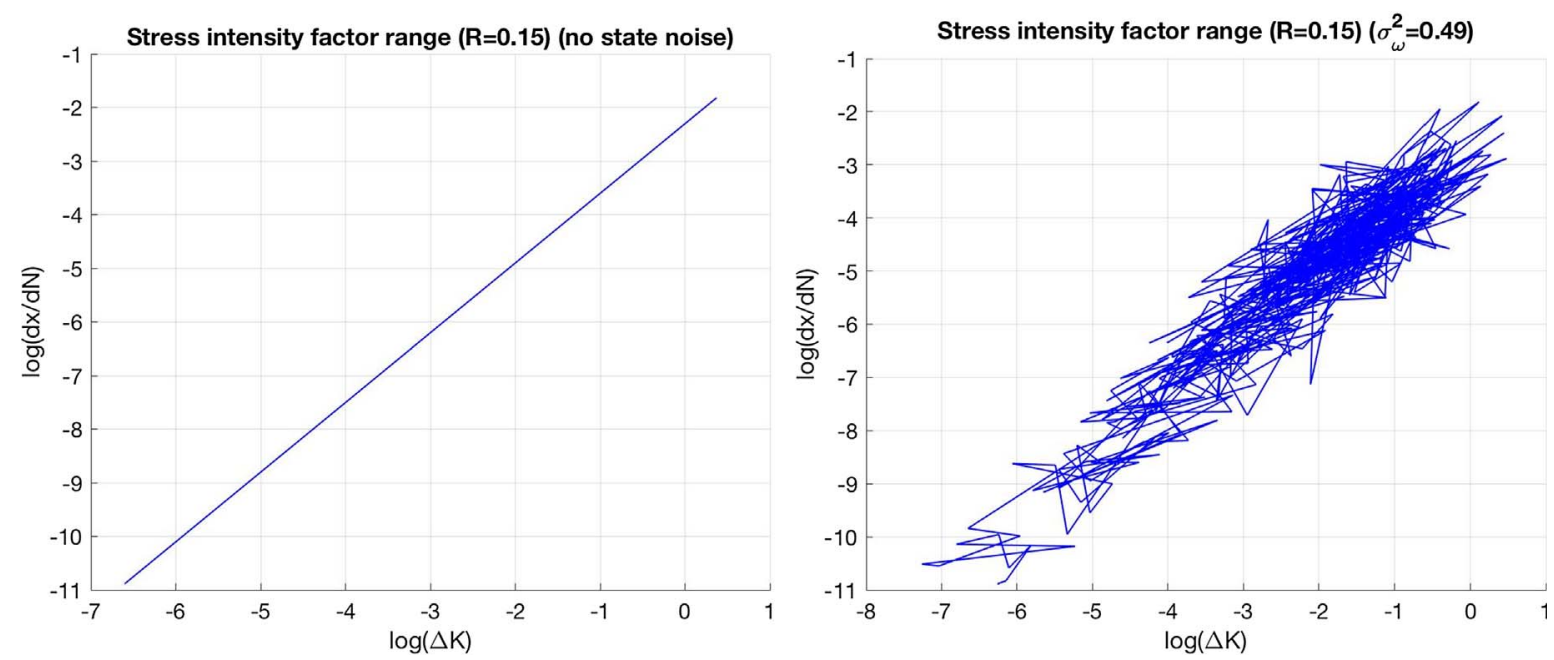

Fig. 4. Stress intensity factor range, $\Delta K \log$ scale, with $R=0.15$. Without state noise considered (left) and with state noise $\sigma_{\omega}^{2}=0.49$ (right). 
(a)
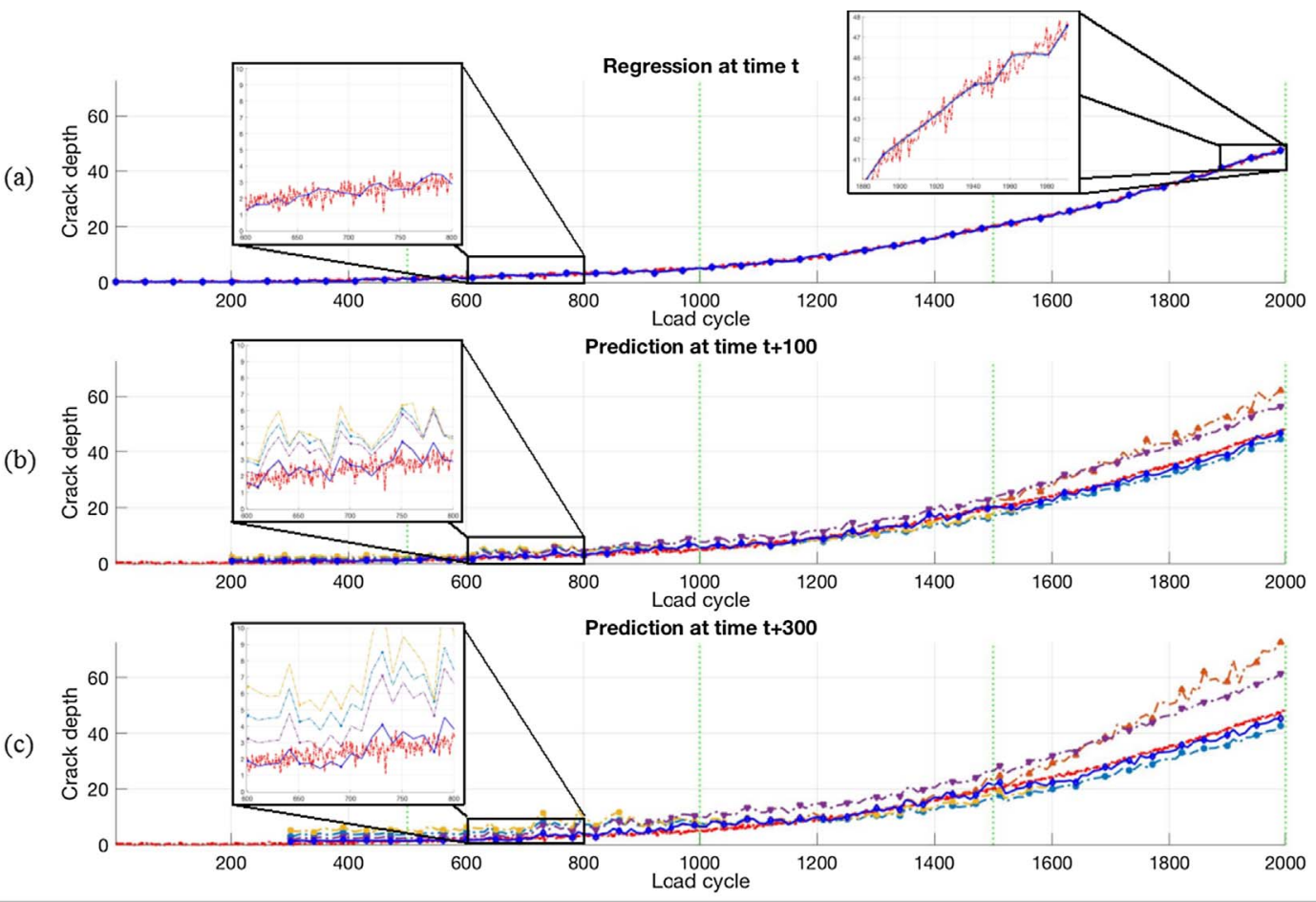

$-\cdot-\cdot$ Measurements $-\cdots \cdot$ Paris-Erdogan model $-\infty$ Polynomial model $\cdots$ Global function-based model $\rightarrow$ Curve fitting function-based model $\rightarrow-$ Proposed ensemble

Fig. 5. Estimation of degradation state at load ratio $\mathrm{R}=0.1$ in three scenarios: (a) Regression at time t; (b) Prediction at time $t+100$; and (c) Prediction at time $\mathrm{t}+300$.

(a)

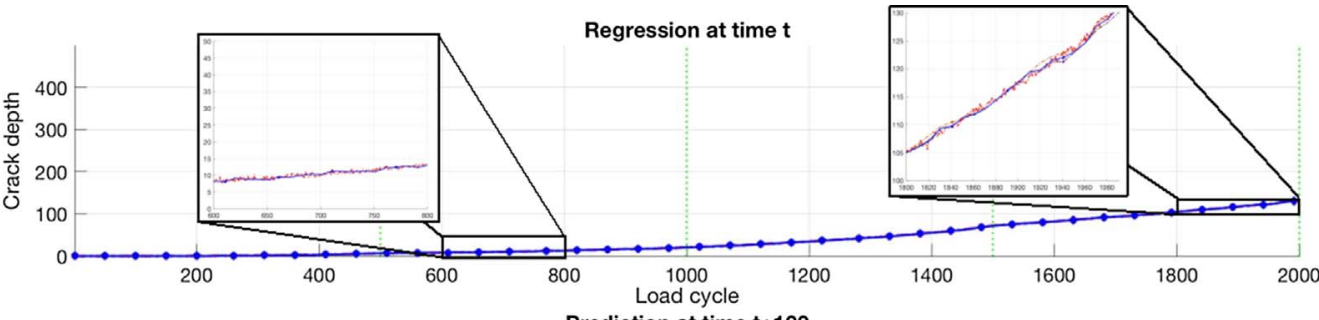

Prediction at time $t+100$

(b)

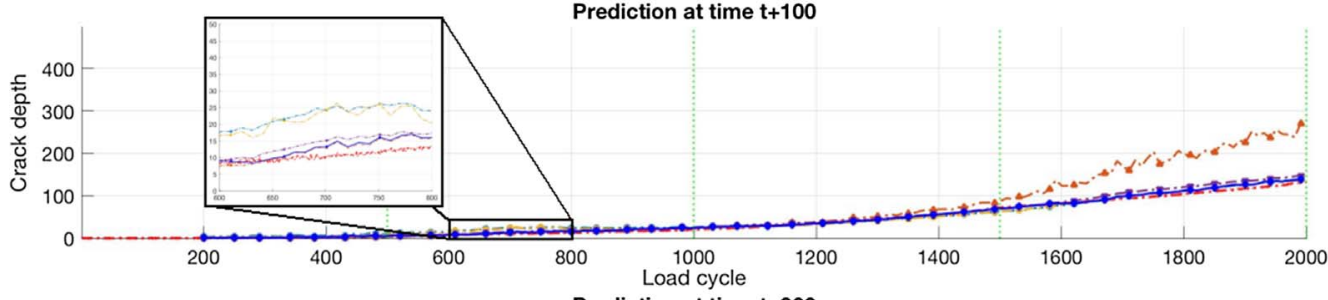

(c)

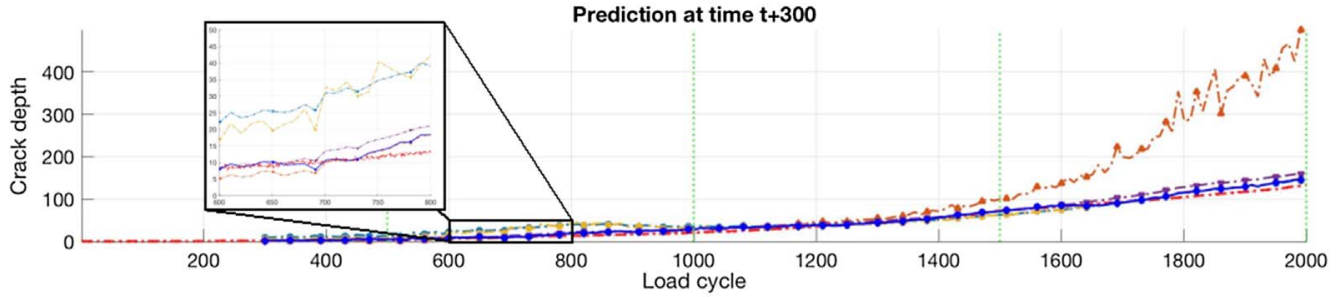

$-\cdot-\cdot$ Measurements $-\leftarrow$ Paris-Erdogan model $-*$ Polynomial model $-\rightarrow$ Global function-based model $-*$ Curve fitting function-based model $\rightarrow-$ Proposed ensemble

Fig. 6. Estimation of degradation state at load ratio $\mathrm{R}=0.15$ in three scenarios: (a) Regression at time $\mathrm{t}$; (b) Prediction at time $\mathrm{t}+100$; and (c) Prediction at time $\mathrm{t}+300$. 
Table 2

MSE results of the degradation state regression and short-term prediction at different load conditions.

\begin{tabular}{|c|c|c|c|c|c|c|}
\hline & \multicolumn{3}{|c|}{$\mathrm{R}=0.1$} & \multicolumn{3}{|c|}{$\mathrm{R}=0.15$} \\
\hline & $\mathrm{t}$ & $\mathrm{t}+100$ & $t+300$ & $\mathrm{t}$ & $t+100$ & $t+300$ \\
\hline Paris-Erdogan model & 0.10 & 1.09 & 12.79 & 0.15 & 10.35 & 151.04 \\
\hline Polynomial function-based model & 0.10 & 4.51 & 60.90 & 0.15 & 278.25 & 9764.72 \\
\hline Global function-based model & 0.10 & 0.69 & 11.92 & 0.15 & 8.94 & 140.21 \\
\hline Curve fitting function-based model & 0.10 & 3.54 & 42.03 & 0.15 & 14.33 & 119.09 \\
\hline Proposed ensemble & 0.10 & 0.38 & 2.07 & 0.12 & 5.23 & 33.14 \\
\hline
\end{tabular}

(a)

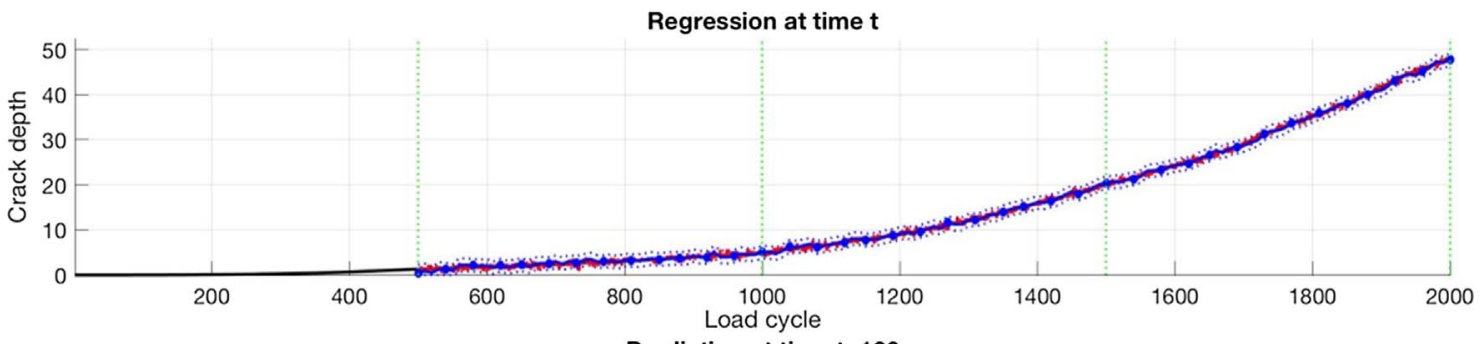

Prediction at time $t+100$

(b)

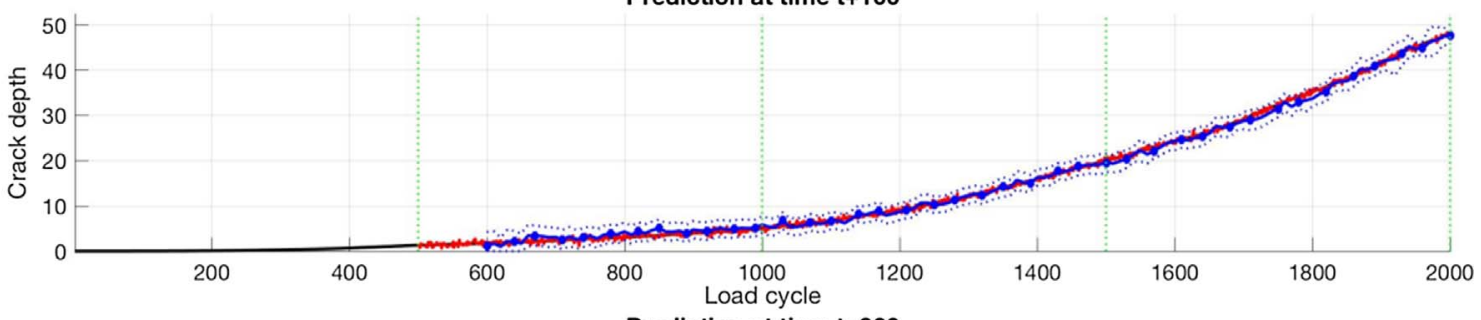

Prediction at time $t+300$

(c)

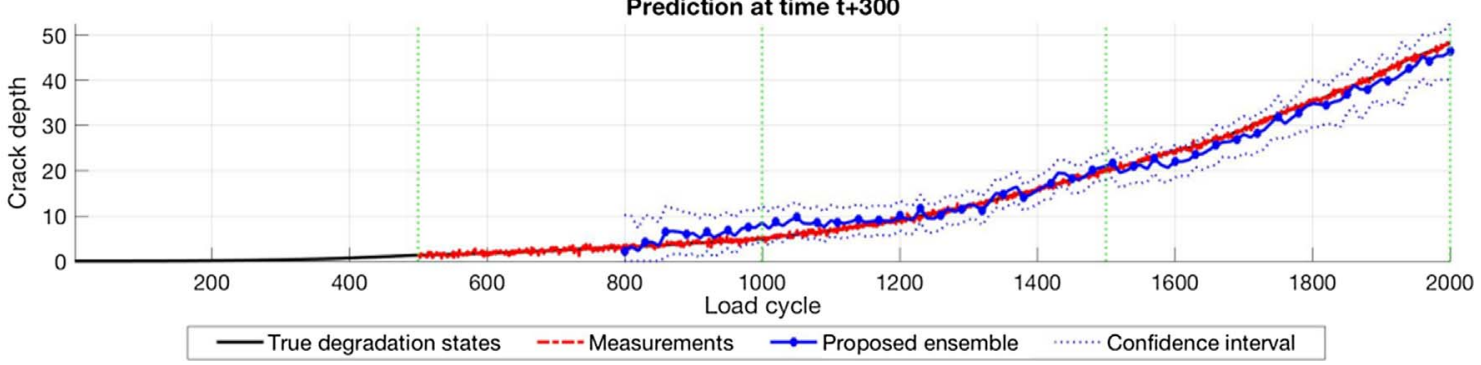

Fig. 7. Estimation of degradation state with measurements that are not available until the time $t_{0}=500$ at load ratio $R=0.1$, in three scenarios: (a) Regression at time $\mathrm{t}$; (b) Prediction at time $\mathrm{t}+100$; and (c) Prediction at time $\mathrm{t}+300$.

$$
x_{t}=x_{t-1}+e^{\omega}\left(\frac{1}{C_{1} x^{m}+C_{2}}\right)(\Delta K)^{m} \Delta t .
$$

\subsection{Dynamic-weighted ensemble for prediction}

Although various stochastic crack growth models have been studied, it is still difficult to develop a unique accurate model for specific degradation processes, particularly for the ones under time-varying operation conditions. In this section, a dynamic-weighted ensemble is presented to find automatically the best combinations of multiple crack growth models with respect to their estimation performances. The proposed algorithm consists of the following three steps:

(a) Step 1: At the load cycle time $t$, when the new measurement is available, the time-varying model parameters and the estimated degradation states given by each model are updated by using the recursive Bayesian algorithm described in Section 2.1.

(b) Step 2: The estimated degradation state of each individual model is weighted by their estimation errors for the last cycles as follows: 
(a)

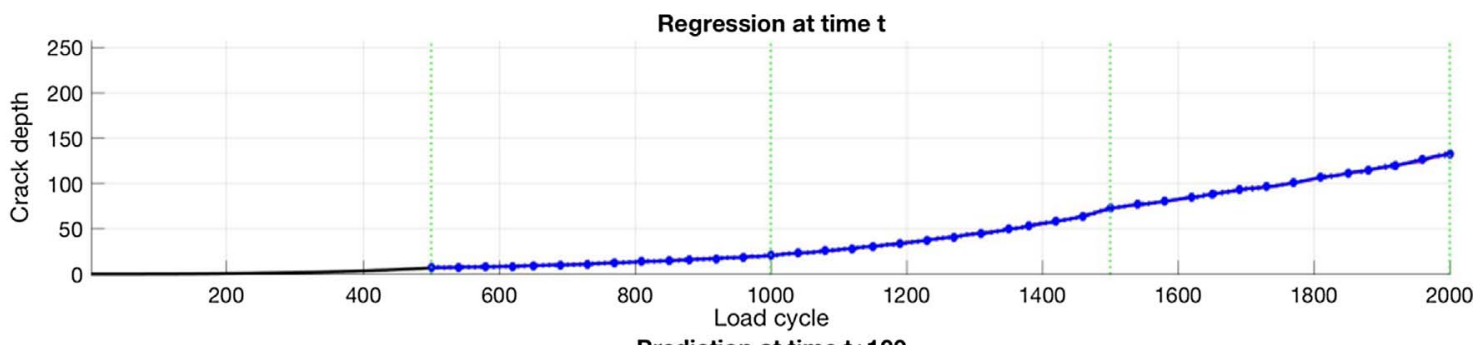

(b)

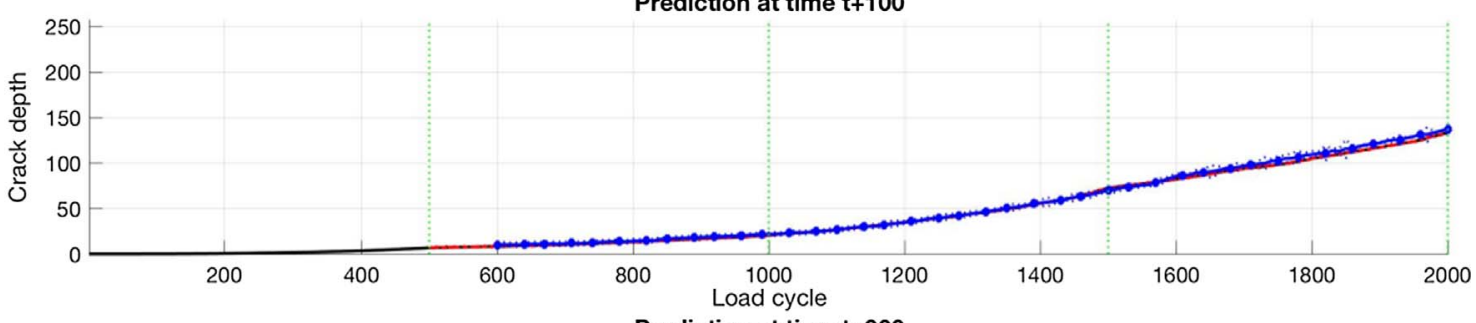

Prediction at time $\mathrm{t}+300$

(c)

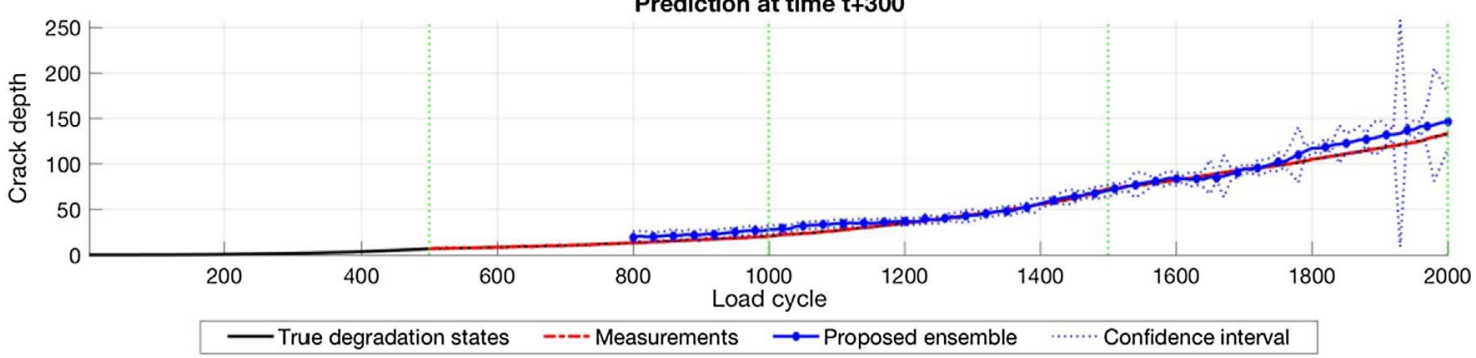

Fig. 8. Estimation of degradation state with measurements that are not available until the time $t_{0}=500$ at load ratio $R=0.15$, in three scenarios: (a) Regression at time $\mathrm{t}$; (b) Prediction at time $\mathrm{t}+100$; and (c) Prediction at time $\mathrm{t}+300$.

$$
w_{t}^{i}=\frac{\left(\phi_{t}^{i}\right)^{-2}}{\sum_{i}\left(\phi_{t}^{i}\right)^{-2}},
$$

where $w_{t}^{i}$ and $\phi_{t}^{i}$ are the dynamic weight and estimation error coefficient of the $i$ th model at time $t$, respectively. The estimation error coefficient $\phi_{t}^{i}$ is defined as follows:

$$
\phi_{t}^{i}=\frac{1}{\delta} \sum_{k=t-\delta}^{t}\left(z_{k}-\hat{x}_{k}^{i}\right)^{2},
$$

where $\delta$ is the time horizon ( $\delta=50$ load cycles in the case study that follows) and $\hat{x}_{k}^{i}$ is the estimated degradation state of the $i$ th model at time $k$. Consequently, the highest weight is given to the model in the ensemble with lowest error at the present time $t$, and vice versa, the smallest weight in constructing the ensemble is assigned to the least accurate model at time $t$.

(c) Step 3: Once the dynamic weights for all models at the current time $t$ are calculated, the predicted degradation state of the ensemble is computed as follows:

$$
\tilde{x}_{T}=\sum_{i}^{N_{M}}\left(\hat{x}_{T}^{i} \times w_{t}^{i}\right),
$$

where $\widetilde{x}_{T}$ is the ensemble predicted state at the load cycle $T$ and $N_{M}$ is the number of degradation models ( $N_{M}=4$ in this study). Note that the weights are for the current load cycle as no new measurements are available for any future load cycles.

\section{Results and discussion}

\subsection{Illustrative case study of fatigue crack growth}

Numerical simulations of fatigue crack growth have been carried out with an initial crack length $x_{0}$ of $10^{-4}$ mm and a test frequency of $1 \mathrm{~Hz}$. The total number of fatigue load cycles is $N=2000$ cycles. To explore the capabilities of the proposed approach under time-varying degradation processes, the fatigue lifetime of the simulated crack growth is split into four continuous and equivalent periods, where the crack length is generated according to different crack growth models as follows: 
(a)

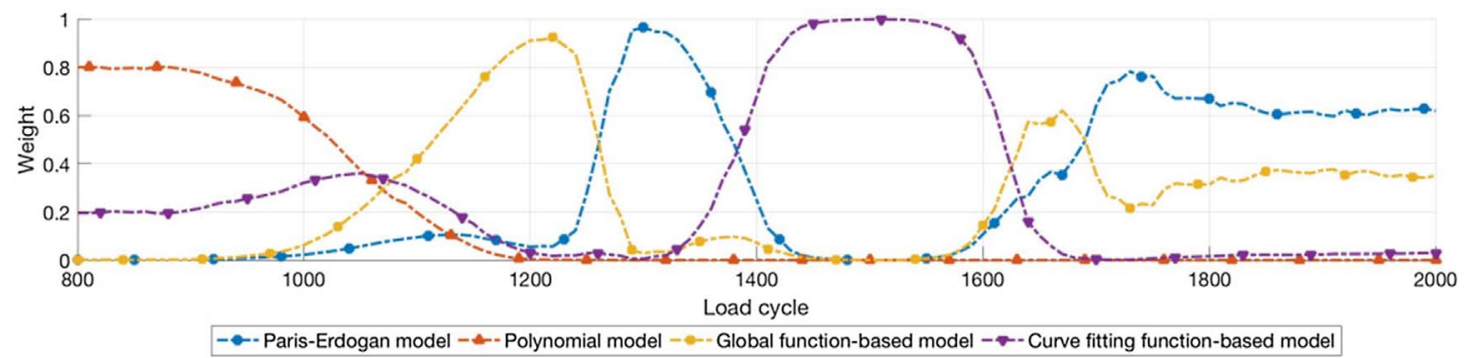

(b)

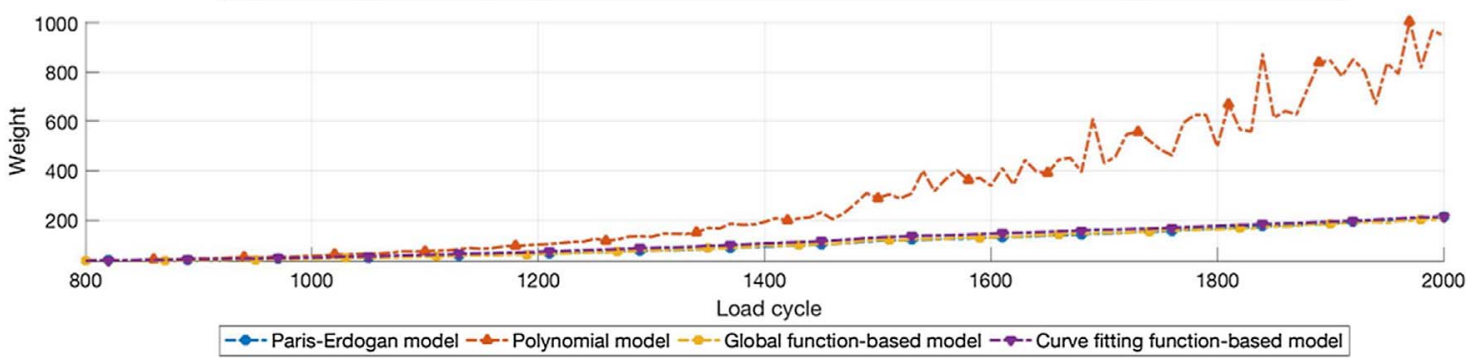

(c)

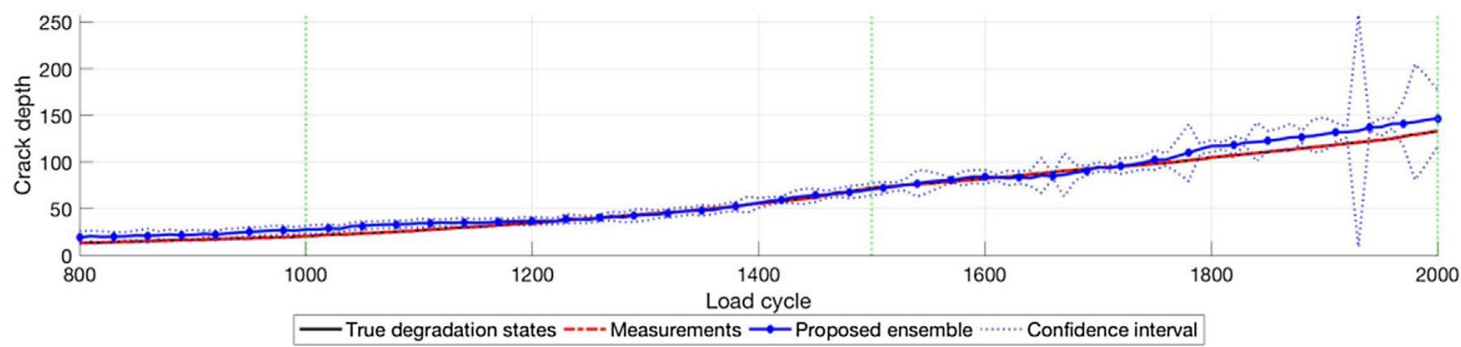

Fig. 9. Degradation state prediction at time $t+300$ with measurements that are not available until the time $t=500$ at load ratio $R=0.15$ : (a) Ensemble weights of individual models; (b) Degradation state prediction of individual models; (c) Degradation state prediction of the proposed ensemble.

(a) In the first 500-load-cycle period, the Paris-Erdogan model is employed to simulate the crack propagation process as described in Eq. (16). In this regard, the Paris-Erdogan model can provide a linear relationship between $\log (d x / d N)$ and $\log (\Delta K)$, in other words, the stress intensity factor (SIF) range of simulated data is compatible to the Region II (Paris region).

(b) In the following period, the polynomial crack growth model in Eq. (18) is used to generate the crack length.

(c) The global function-based crack growth model in Eq. (21) is, then, employed for the third period.

(d) Finally, in the last period, from the cycle 1501 to 2000, the curve fitting function-based crack growth model in Eq. (23) is utilized.

The model parameters in this work are first initialized based on empirical knowledge, as detailed in Table 1. Subsequently, a Bayesian-based parameter identification approach, as presented in Section 2.1.2, is applied to adaptively update the parameters according to the real-time information from measured data at each load cycle.

For the purpose of extensively validating the effectiveness of the proposed approach for drifting degradation processes, two crack growth profiles under different conditions of load ratio, $R=0.1$ and $R=0.15$, are artificially integrated to expand the case study of the simulated crack growth, as illustrated in Fig. 2. Specifically, the load ratio $R$, or the stress ratio, measures the general influence of the mean stress on the fatigue crack growth behavior, and is defined by the ratio of the minimum to the maximum stresses experienced during a cycle. As the ratio $R$ increases, the fatigue crack growth rate curve is shifted toward higher $d x / d N$ [38].

In order to validate the SIF ranges of the generated crack growth data, an investigation is carried out on the first 500-load-cycle period, where the crack size is very small, and the results are illustrated in Figs. 3 and 4. The state noise is also considered in this investigation. As expected, the results clearly show a linear relationship between $\log (d x / d N)$ and $\log (\Delta K)$ in all cases of stress ratio conditions, which means that the simulated data completely correspond to the Region II (Paris region).

\subsection{Performance evaluation}

The proposed prognostic framework based on recursive Bayesian technique and dynamic-weighted ensemble is applied to determine the best combination of multiple crack growth models in terms of degradation prediction performance. More specifically, when a new monitored measurement is available, the degradation states and crack growth model parameters are estimated online via the Bayesian technique. The four stochastic crack growth models described in Section 2.2 are considered. The weight for each individual model is updated at the current load cycle and the ensemble is obtained by integrating the individual degradation models. The mean square error (MSE) is considered as the performance evaluation index to indicate prediction accuracy: 

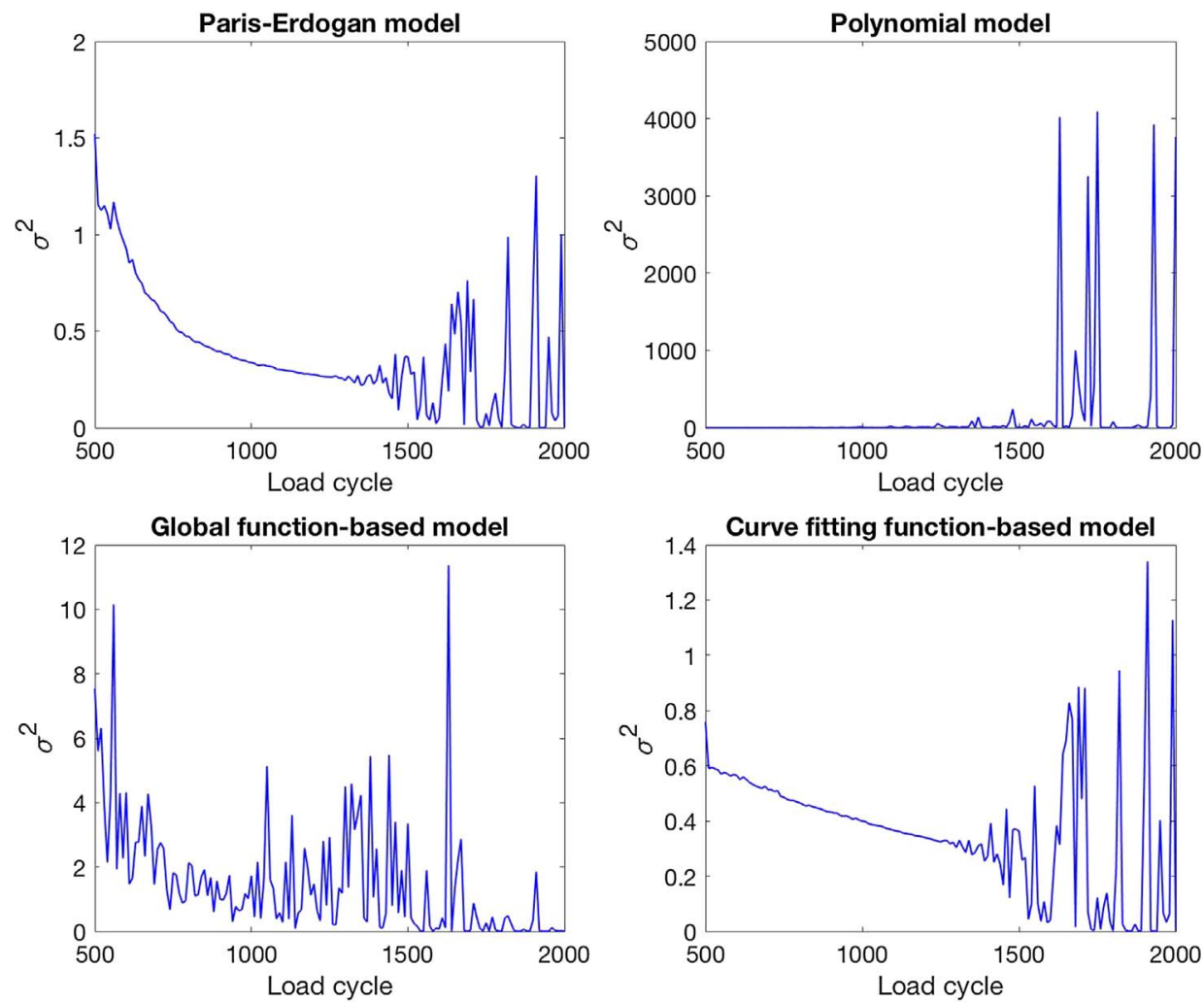

Fig. 10. Variances of degradation state prediction at time $t+300$ with measurements that are not available until the time $t_{0}=500$ at load ratio $R=0.15$.

$$
M S E_{i}=\frac{1}{N} \sum_{t=1}^{N}\left(x_{t}-\hat{x}_{t}^{i}\right)^{2},
$$

where $x_{t}$ and $\hat{x}_{t}^{i}$ denote the true degradation state and the prediction of the ith crack growth model at time $t$, respectively.

The degradation state estimations at different load ratios are shown in Figs. 5 and 6 . As expected, the estimated degradation states among different models are similar for the current time, as shown in Figs. 5(a) and 6(a), but obviously separated for short-term state predictions. This indicates the effectiveness of the prediction error-based dynamic weights in reflecting the performance of different degradation models. An interesting observation in Figs. 5 and 6 is that the polynomial model can exhibit satisfactory performance when the fatigue crack depth is small, but its performance is rapidly degraded when the crack becomes longer. This is mainly because of the fact that the polynomial function only fits in the linear least square stage of the degradation process, as mentioned in Section 2.2.2. In other words, the polynomial model is able to achieve satisfactory performance only for linear and deterministic fatigue crack growth processes. In contrast, by combining dynamically different degradation models, the proposed ensemble approach can achieve superior performance to the individual models in predicting the degradation states of fatigue crack growth. Table 2 shows that the proposed ensemble framework outperforms the individual crack growth models, yielding a prediction accuracy of 2.07 and 33.14 in terms of MSE for short-term degradation state prediction at time $t+300$ under the load ratios $R=0.1$ and $R=0.15$, respectively.

The impact of uncertainty on the performance of the proposed framework in case of unknown initial state of degradation has also been investigated. In this case, the monitored data are assumed unavailable from time 1 to $t_{0}\left(t_{0}=500\right.$ in this study), and the true degradation state of the system $x_{t_{0}}$ is also assumed unknown. The performance of the proposed approach under different load ratio conditions is shown in Figs. 7 and 8. The dashed line with marker is the predicted degradation states of the proposed approach while the dotted lines are the $95 \%$ confidence intervals. The results in Figs. 7 and 8 show that the proposed framework can yield accurate state predictions even without knowledge of the initial degradation state. In Fig. 8(c), some abnormal spikes in the confidence intervals can be observed. Note that these abnormal spikes exist only in the case study in which the degradation state prediction is performed at time $t+300$ with no available measurement until the time $t_{0}=500$. For the relative shorter prediction horizon, no spike is observed. This can be explained by the fact that for a long-term prediction time $t+300$, the performances of individual models in the ensemble can be unexpectedly degraded due to the propagation of uncertainty. As can be seen in Figs. 9 and 10 below, in the last 400 load cycles, the performance diversity between the polynomial model and the others is clearly observed, and, furthermore, the variance of the polynomial model also rapidly increases, resulting in unsatisfactory performance in the estimation of the confidence intervals. 


\section{Conclusions}

This paper proposes a prognostic framework for predicting the degradation states of fatigue crack growth under different load conditions. Although several degradation models have been investigated for fatigue crack growth prognosis in the literature, there is no general consensus on a comprehensive crack growth model over different degradation processes. To address this issue, a dynamicweighted ensemble of multiple degradation models is presented. The key idea of the proposed approach is to utilize a dynamic weight vector, which is updated at each load cycle when the new measurements become available, for evaluating individual degradation models performance with respect to their estimation errors on previous cycles. Short-term predictions of crack growth are also provided to anticipate and proactively prevent sudden breakdowns of the component in a near future. Simulation results show that the proposed prognostic framework can yield a satisfactory performance under evolving operating conditions, and outperforms individual models for fatigue crack growth in terms of prediction accuracy.

The performance of the proposed dynamic-weighted ensemble framework depends also on the specific degradation models used in the ensemble. More advanced degradation models of fatigue crack growth (e.g. those of the FKM Directive [39]) can be accommodated in the framework. Moreover, not only state-dependent but also age-dependent models can be used for predicting the degradation process of fatigue crack growth [40]. Note that the predetermined initial values of the model parameters may affect the prediction performance: their values should be initialized based on the available measurements of the equipment. To address this issue, research on dynamically initializing the model parameters, e.g. by using the Maximum Likelihood Estimation (MLE) [41], can be considered in future work.

\section{References}

[1] Eckmann S, Schweizer C. Characterization of fatigue crack growth, damage mechanisms and damage evolution of the nickel-based superalloys MAR-M247 CC (HIP) and CM-247 LC under thermomechanical fatigue loading using in situ optical microscopy. Int J Fatigue 2017;99:235-41.

[2] Kamaya M. Fatigue crack tolerance design for stainless steel by crack growth analysis. Eng Fract Mech 2017;177:14-32.

[3] Lee S, Zi G, Mun S, Kong J, Choi J. Probabilistic prognosis of fatigue crack growth for asphalt concretes. Eng Fract Mech 2015;141:212-29.

[4] Zhao Z, Haldar A, Breen F. Fatigue-reliability evaluation of steel bridges. J Struct Eng 1994;120(5):1608-23.

[5] Haile M, Riddick J, Assefa A. Robust particle filters for fatigue crack growth estimation in rotorcraft structures. IEEE Trans Reliab 2016;65(3):1438-48.

[6] Haile M, Chen T, Shiao M, Le D. Crack growth behavior in preloaded metallic nested-angle plates under flight load spectrum. In: Proceedings of the 2011 annual conference on experimental and applied mechanics, vol. 6, 2011. p. 3-11.

[7] Urban M. Analysis of the fatigue life of riveted sheet metal helicopter airframe joints. Int J Fatigue 2003;25(9-11):1013-26.

[8] Yang H, Doquet V, Zhang Z. Fatigue crack growth in two TWIP steels with different stacking fault energies. Int J Fatigue 2017;98:247-58.

[9] Mayén J, Abúndez A, Pereyra I, Colín J, Blanco A, Serna S. Comparative analysis of the fatigue short crack growth on Al 6061-T6 alloy by the exponential crack growth equation and a proposed empirical model. Eng Fract Mech 2017;177:203-17.

[10] Yang G, Pointeau V, Tevissen E, Chagnes A. A review on clogging of recirculating steam generators in Pressurized-Water Reactors. Prog Nucl Energy 2017;97:182-96.

[11] Prusek T, Moleiro E, Oukacine F, Adobes A, Jaeger M, Grandotto M. Deposit models for tube support plate flow blockage in Steam Generators. Nucl Eng Des 2013;262:418-28.

[12] Compare M, Bellani L, Zio E. Availability model of a PHM-equipped component. IEEE Trans Reliab 2017;66(2):487-501.

[13] Amiri M, Modarres M, Droguett E. AE entropy for detection of fatigue crack initiation and growth. In: IEEE conference on prognostics and health management (PHM); 2015, Austin, TX. p. 1-8.

[14] Cadini F, Zio E, Avram D. Model-based Monte Carlo state estimation for condition-based component replacement. Reliab Eng Syst Saf 2009;94(3):752-8.

[15] Amiri M, Modarres M. Short fatigue crack initiation and growth modeling in aluminum 7075-T6. Proc Inst Mech Eng Part C: J Mech Eng Sci 2014;229(7):1206-14.

[16] Xu J, Wang Y, Xu L. PHM-oriented integrated fusion prognostics for aircraft engines based on sensor data. IEEE Sens J 2014;14(4):1124-32.

[17] Tse P, Atherton D. Prediction of machine deterioration using vibration based fault trends and recurrent neural networks. J Vib Acoust 1999;121(3):355.

[18] Tobon-Mejia D, Medjaher K, Zerhouni N, Tripot G. A data-driven failure prognostics method based on mixture of Gaussians Hidden Markov models. IEEE Trans Reliab 2012;61(2):491-503.

[19] Huang R, Xi L, Li X, Richard Liu C, Qiu H, Lee J. Residual life predictions for ball bearings based on self-organizing map and back propagation neural network methods. Mech Syst Sig Process 2007;21(1):193-207.

[20] Wang Z, Wang W, Hu C, Si X, Li J. A real-time prognostic method for the drift errors in the inertial navigation system by a nonlinear random-coefficient regression model. Acta Astronaut 2014;103:45-54.

[21] Gebraeel N, Pan Jing. Prognostic degradation models for computing and updating residual life distributions in a time-varying environment. IEEE Trans Reliab 2008;57(4):539-50.

[22] Zárate B, Caicedo J, Yu J, Ziehl P. Bayesian model updating and prognosis of fatigue crack growth. Eng Struct 2012;45:53-61.

[23] Orchard M. A particle filtering-based framework for online fault diagnosis and failure prognosis. Ph.D. Thesis, Department of Electrical and Computer Engineering, Georgia Institute of Technology; 2007.

[24] Bogdanoff J, Kozin F. Probabilistic models of cumulative damage. New York: Wiley; 1985.

[25] Sobczyk K, Spencer B. Random fatigue. Boston: Academic Press; 1992.

[26] Wu W. On the Markov approximation of fatigue crack growth. Probab Eng Mech 1986;1(4):224-33.

[27] Rocha M, Schuëller G. A probabilistic criterion for evaluating the goodness of fatigue crack growth models. Eng Fract Mech 1996;53(5):707-31.

[28] Yang J, Manning S. Stochastic crack growth analysis methodologies for metallic structures. Eng Fract Mech 1990;37(5):1105-24.

[29] Yang J, Manning S. A simple second order approximation for stochastic crack growth analysis. Eng Fract Mech 1996;53(5):677-86.

[30] Hossein S, Mohammad P, Saeid K. Assessment of stochastic fatigue failures based on deterministic functions. In: 13th international conference on probabilistic safety assessment and management (PSAM 13), Seoul, Korea; 2016.

[31] Wu W, Ni C. Probabilistic models of fatigue crack propagation and their experimental verification. Probab Eng Mech 2004;19(3):247-57.

[32] Xing Y, Ma E, Tsui K, Pecht M. An ensemble model for predicting the remaining useful performance of lithium-ion batteries. Microelectron Reliab 2013;53(6):811-20.

[33] Su X, Wang S, Pecht M, Zhao L, Ye Z. Interacting multiple model particle filter for prognostics of lithium-ion batteries. Microelectron Reliab 2017;70:59-69.

[34] Paris P, Erdogan F. A critical analysis of crack propagation laws. J Basic Eng 1963;85(4):528.

[35] Irwin G. Analysis of stresses and strains near the end of a crack traversing a plate. J Appl Mech 1957;24:361-4.

[36] Myötyri E, Pulkkinen U, Simola K. Application of stochastic filtering for lifetime prediction. Reliab Eng Syst Saf 2006;91(2):200-8.

[37] Ni C. Formulation of a polynomial stochastic fatigue crack growth model. Adv Mater Res 2014;909:467-71.

[38] Gdoutos E. Fracture mechanics. Dordrecht: Springer; 2005.

[39] FKM-Guideline. Fracture mechanics proof of strength for engineering components. VDMA Publ., 1st ed. [2001, in German], 2nd ed. [2004, in German and English], 3rd ed. [2005, in German].

[40] Li N, Lei Y, Guo L, Yan T, Lin J. Remaining useful life prediction based on a general expression of stochastic process models. IEEE Trans Ind Electron 2017;64(7):5709-18.

[41] Lei Y, Li N, Gontarz S, Lin J, Radkowski S, Dybala J. A model-based method for remaining useful life prediction of machinery. IEEE Trans Reliab 2016;65(3):1314-26. 